\title{
Acupuncture alleviates the affective dimension of pain in a rat model of inflammatory hyperalgesia
}

\author{
Yu Zhang ${ }^{1,2}$, Xianze Meng ${ }^{1,3}$, Aihui Li ${ }^{1}$, Jiajia Xin ${ }^{1}$, Brian M. Berman ${ }^{1}$, Lixing Lao ${ }^{1}$, Ming \\ Tan $^{4}$, Ke Ren $^{5}$, and Rui-Xin Zhang ${ }^{1, *}$ \\ ${ }^{1}$ Center for Integrative Medicine, School of Medicine, University of Maryland, Baltimore, MD \\ 21201 USA \\ ${ }^{2}$ Department of Neurobiology, Shanxi Medical University, Taiyuan 030001, Shanxi, P. R. China \\ ${ }^{3}$ Department of Traditional Chinese Medicine, Shanghai Changzheng Hospital, Second Military \\ Medical University, Shanghai, 200001, P. R. China \\ ${ }^{4}$ Division of Biostatistics, University of Maryland Greenebaum Cancer Center, Baltimore, MD \\ 21201 USA \\ ${ }^{5}$ Department of Neural and Pain Sciences, Dental School, University of Maryland, Baltimore, MD \\ 21201 USA
}

\begin{abstract}
Although studies demonstrate that electroacupuncture (EA) alleviates the sensory dimension of pain, they have not addressed EA's effect on the affective dimension. An inflammatory pain rat model, produced by a complete Freund adjuvant (CFA) injection into the hind paw, was combined with a conditioned place avoidance (CPA) test to determine EA's effects and its underpinning mechanism on the affective dimension of pain. CFA-injected rats showed place aversion, i.e. the affective dimension of pain, by spending less time in a pain-paired compartment after conditioning than before, while saline-injected rats did not. CFA rats given EA treatment at GB30 before a postconditioning test showed no aversion to the pain-paired compartment, indicating that EA inhibited the affective response. Intra-rostral anterior cingulate cortex (rACC) administration of a $\kappa-$, but not $\mu$-opioid receptor antagonist, blocked EA action. These data demonstrate that EA activates opioid receptors in the rACC to inhibit the affective dimension of pain.
\end{abstract}

\section{Keywords}

acupuncture; opioid; anterior cingulate cortex; pain; complete Freund's adjuvant

\section{Introduction}

Pain has both sensory-discriminative and emotional-affective dimensions. In the past few decades, persistent pain animal models have been used to study pain mechanisms and the analgesic activity of drugs. These studies have used nocifensive reflexes, including spinal reflexes (tail flick, paw withdrawal), spino-bulbospinal reflexes (jumping, abdominal stretching), and simple innate behaviors (vocalization, scratching, biting, licking, guarding) to investigate the sensory dimension of pain. Recently, a conditioned place avoidance (CPA) test has been used as an indirect assessment of the affective component of pain [1]. Studies

*Corresponding author: Dr. Rui-Xin Zhang, Center for Integrative Medicine, 685 W. Baltimore street, MSTF Rm 8-22, Baltimore, MD 21201, Telephone: 410-706-1582, Fax: 410-706-1583, Rzhan001@umaryland.edu. 
have demonstrated that low dosage aspirin attenuates escape/avoidance behavior in a rodent model of inflammatory pain [2]. Moreover, low dosage celecoxib, diclofenac, and duloxetine significantly inhibit the avoidance response associated with stimulation of an injured paw [3]. These studies demonstrate that it is possible to study the affective dimension of pain indirectly in an animal model.

Acupuncture, a traditional therapeutic modality, has been used in China and other Asian countries for thousands of years to treat a variety of diseases and symptoms, including pain [4]. Previous studies show that EA alleviates the sensory-discriminative dimension of pain. For instance, EA has produced significant recovery in stepping force in an ankle sprain pain model [5] and significant increase in hind paw withdrawal latency (PWL) in inflammatory pain animal models [6, 7]. Further, in an uncontrolled observation, acupuncture improved the affective dimension of a variety of chronic pain conditions, including headache, facial, and spine-associated pain syndromes [8]. While the study suggested that acupuncture may inhibit the affective dimension of pain, the underlying mechanisms were not explored.

EA's effects and its underpinning mechanism on the affective dimension of pain was studied by combining a complete Freund adjuvant (CFA) injection-induced inflammatory pain rat model with a CPA test $[1,9]$. Previous studies suggest that the anterior cingulate cortex (ACC) plays a role in the affective dimension of pain. In animals, bilateral ACC lesions decrease the development of place-avoidance behavior in a formalin-conditioned place avoidance (CPA) model [1]. ACC lesions in rats with nerve injury also significantly decrease escape/avoidance behavior but do not alter mechanical hypersensitivity [10]. Since it has been well demonstrated that acupuncture induces release of endogenous opioids [11], we hypothesized that rostral ACC (rACC) opioids underpin acupuncture's inhibition of the affective dimension of pain.

\section{Experimental procedures}

\section{Animal Preparation}

Male Sprague-Dawley rats weighing 250-275 g (Harlan, Indianapolis, IN) were kept under controlled laboratory conditions $\left(22^{\circ} \mathrm{C}\right.$, relative humidity $40 \%-60 \%, 12$-hour alternate light-dark cycles, food and water ad libitum) and were acclimatized to the environment for 5 days prior to an experimentation. The animal protocols were approved by the Institutional Animal Care and Use Committee (IACUC) of the University of Maryland School of Medicine, Maryland, USA.

\section{Experimental design}

Three experiments were conducted.

In Experiment 1, rats were divided into two groups ( $\mathrm{n}=8$ / group): 1) saline and 2) CFA. Saline or CFA $(0.08 \mathrm{ml})$ was injected into one hind paw. The aim of this experiment was to confirm whether the apparatus chambers produce neutral stimuli and whether CFA-induced spontaneous pain generates a negative affective response that can be assessed with CPA.

In Experiment 2, CFA-injected rats were divided into four groups (n=8 per group): 1) saline + sham EA control, 2) saline + EA treatment, 3) CFA + sham EA control, and 4) CFA + EA treatment. EA was given to rats before a post-conditioning test on day three to determine whether EA inhibits CFA-induced CPA,

In Experiment 3, to investigate the involvement of opioid receptors in EA action, rats were prepared for bilateral intra-rACC cannulation and allowed to recover for five days prior to experiment. Four groups of saline- and CFA-injected rats were treated with EA or sham EA: 
1) saline + sham EA, 2) CFA + sham EA, 3) saline + EA, and 4) CFA + EA. The rats in Group 4, CFA + EA, were randomly divided into three subgroups ( $n=8$ per group) to reveal the role of endogenous opioids in the EA inhibition of affective response: 1) $6.25 \mathrm{nmol} / 0.25$ $\mu 1 /$ side of the $\mu$-opioid receptor antagonist D-Phe-Cys-Tyr-D-Trp-Orn-Thr-Pen-Thr amide (CTOP) + EA prior to, 2) $10 \mathrm{nmol} / 0.4 \mu \mathrm{l} / \mathrm{side}$ of the $\kappa$-opioid receptor antagonist norbinaltorphimine (nor-BNI) + EA, and 3) vehicle + EA. The antagonists, dissolved in saline, were administered five min before an EA treatment prior to a post-conditioning test on day three. The dosages are based on our preliminary study.

Groups 1-3, saline + EA, CFA + sham EA, and saline + sham EA, were similarly divided into three subgroups and tested.

\section{EA treatment}

EA treatment was performed according to the procedures previously developed in our laboratory [7]. The animals were gently handled for $30 \mathrm{~min}$ each day for two or three days before the experiment. After cleaning a rat's skin with alcohol swabs, one investigator held the animal while the second swiftly inserted a disposable acupuncture needle (gauge \#32, $0.5 \mathrm{in}$. in length), with electrodes soldered to its handle, approximately $1 / 2$ in. deep into each flank at the equivalent of human acupoint GB30. In humans, GB30, the 30th point on the Gallbladder Meridian, is located at the junction of the lateral third and medial two-thirds of the distance between the greater trochanter and the hiatus of the sacrum. The needles and electrodes were stabilized with adhesive tape. The procedure typically lasted less than $20 \mathrm{~s}$ and caused little distress to the animal.

EA was delivered by an electrical stimulator via an isolator (A360D Stimulus Isolator, World Precision Instruments) that converts electrical voltage into constant electrical current. In order to minimize discomfort to the animal, the current was adjusted slowly over the period of approximately two min to the designated level of $3 \mathrm{~mA}$, the maximum intensity the animal can tolerate without distress. The stimulation was administered to the muscles, not directly to the peripheral nerve. Mild muscle twitching was observed at this intensity.

EA treatment was given $2 \mathrm{~h} 20 \mathrm{~min}$ before the post-conditioning test on day three. Twenty min of stimulation at $10 \mathrm{~Hz}, 3 \mathrm{~mA}, 0.1 \mathrm{~ms}$ pulse width was applied bilaterally at GB30, once at the beginning and again at the end of a $2 \mathrm{~h}$ period [7]. During EA treatment, each rat was placed on a glass surface under an inverted clear plastic chamber (approximately $5^{\prime \prime} \times$ $\left.8^{\prime \prime} \times 11^{\prime \prime}\right)$ without restraints or anesthetics. The animals remained awake and still during treatment, and no signs of distress were observed [7]. For sham EA control, acupuncture needles were inserted bilaterally at acupoint GB30; a pair of electrodes from the stimulator was attached to the ends of the needles but no electrical current was delivered. It should be noted that this control is different from regular acupuncture, in which needles are manually or electrically stimulated to alleviate pain.

\section{CPA test}

Place conditioning was performed as described by Fenu et al. [12], with modifications. The place conditioning apparatus is made of Plexiglas and consists of two equal, rectangular compartments $\left(12^{\prime \prime}\right.$ long $\times 8^{\prime \prime}$ high $\times 5^{\prime \prime}$ wide) positioned on top of mesh screens and separated by guillotine doors. One compartment is covered on three sides and the ceiling with horizontal yellow 0.5 -inch stripes at 0.5 -inch intervals; its bottom is \#8×8 plain steel mesh ( $8 \mathrm{~mm}$ holes). The other compartment is covered on three sides and the ceiling with vertical orange stripes and has $\# 3 \times 3$ plain steel mesh $(3 \mathrm{~mm}$ holes) on its floor. The guillotine doors are covered with the colored stripes corresponding to their respective walls; they were inserted during conditioning sessions and removed during pre- and post- 
conditioning tests. The vertically striped compartment is laterally illuminated on the outside with a Coast Led Lenser mini-Tac Torch. The colored horizontal and vertical stripes and the light are the visual cues; the differing steel mesh is the tactile cue. The testing room has dim indirect lighting consisting of one $15-\mathrm{W}$ bulb positioned about $1 \mathrm{~m}$ from the apparatus. The apparatus was cleaned with $75 \%$ ethanol after each test.

On day one, baseline time spent by the rats in each of the two distinct compartments during a 10-min preconditioning period was recorded $[1,12]$. The animal was considered to be in a chamber when both its front paws were in it. On day two, each rat was free to explore one of the conditioning compartments for $30 \mathrm{~min}$. Thirty min later, saline or CFA (Sigma, St Louis, MO; $0.08 \mathrm{ml}, 40 \mu \mathrm{g}$ Mycobacterium tuberculosis), suspended in an 1:1 oil/saline emulsion, was subcutaneously injected into the plantar surface of one hind paw using a 25-gauge hypodermic needle [13]. Two $h$ after the CFA injection, the rat was free to explore the second compartment for another $30 \mathrm{~min}$. The pain-paired compartment was alternated so that half of the subjects were pain-conditioned in the orange chamber, the other half in the yellow. During the10-min post-conditioning test on day three, the time spent by the rats in each compartment was recorded again (Fig. 1). The investigator who performed the CPA test was blinded to group assignment.

\section{Intra-Bilateral Cingulate Cortex Cannulation}

Animals were anesthetized with sodium pentobarbital $(50 \mathrm{mg} / \mathrm{kg}$, i.p.) and held in a stereotaxic frame (Stoelting, Wood Dale IL). An incision was made on the midline of the head and a small hole was drilled. A double, bilateral 26-gauge stainless steel guide cannula (Plastic One, Roanoke, VA) was implanted toward the ACC, $2.6 \mathrm{~mm}$ rostrally, $0.6 \mathrm{~mm}$ laterally, and $2.4 \mathrm{~mm}$ ventrally to the bregma according to the Paxinos and Watson flat skull coordinate system. This double guide cannula was secured with dental cement and two small screws. A double, bilateral dummy cannula, cut to extend $0.5 \mathrm{~mm}$ beyond the guide cannula and covered with a dust cap, remained in the guide except during drug infusion. Following cannulation, animals were housed singly and allowed to recover for five days prior to the experiment.

For drug infusion, a $0.6 \mathrm{~cm}$ length of PE-50 tubing was connected to each end of a $15-\mathrm{cm}$ length of PE-10 tubing. During infusion, the dummy cannula was replaced by an injector that was inserted $0.5 \mathrm{~mm}$ beyond the guide cannula to target the ACC. One end of the tubing was connected to the injector and the other, to a 50- $\mu 1$ Hamilton syringe. The solution was infused at $0.1 \mu \mathrm{l} / \mathrm{min}$ for a total of $0.25 \mu \mathrm{l}$ on each side of the rACC with a pump (KD Scientific, Model 780210). After infusion, the injector was left in the cannula for another 2 min to allow the chemicals to spread at the injected area.

\section{Histology}

After the experiment, the infusion site was verified by histology. The animals were perfused with saline and $10 \%$ formalin under analgesia with sodium pentobarbital. The brains were removed and immersed in 10\% formalin for $2 \mathrm{~h}$ and transferred to $30 \%$ sucrose. The tissue at the cannula site was cut into $40-\mu \mathrm{m}$ thick coronal sections which were stained and examined under a microscope to determine the location of the cannula according to Paxinos and Watson's atlas.

\section{Statistical analysis}

CPA score magnitudes (Figs. $3 \& 4 \mathrm{D}$ ), used as an indicator of affective response, were determined by subtracting pre-conditioning time spent by each rat in the pain-paired compartment from the post-conditioning time it spent there, then calculating the average time of each group of rats. The data were analyzed with ANOVA to reveal whether CFA- 
induced pain produced an affective response, whether EA inhibited such a response, and whether opioid receptors were involved in the EA effect. Bonferroni post-tests were conducted to reveal differences among groups of rats (GraphPad Prism 5.0). $\mathrm{P}<0.05$ was considered significant.

\section{Results}

\section{Hind-paw CFA injection induced CPA}

During the 10-min preconditioning test, saline- and CFA-injected rats spent similar amounts of time in the two compartments, indicating no preference for either. But when a hind-paw CFA injection was paired with a particular compartment, rats spent less time in that compartment during the post-conditioning test than during preconditioning, demonstrating place aversion to the compartment (Fig. 2, columns 3 vs 4). By contrast, saline-injected rats showed no aversion to the saline-paired compartment (Fig. 2, columns 1 vs 2). These results indicate that the apparatus chambers produced neutral stimuli and confirmed that a CFA injection induced pain affect compared to saline-injection control.

\section{EA treatment inhibited CFA-induced CPA}

During the preconditioning test, rats spent similar amounts of time in the two compartments, indicating no preference for either. When a hind paw CFA injection was paired with a particular compartment, rats receiving sham EA spent less time there during the postconditioning test than during preconditioning, demonstrating place aversion to that compartment (Fig. 3 column 1). By contrast, EA-treated rats showed no aversion to the painpaired compartment (Fig. 3, column 2). These results demonstrate that EA treatment inhibited CFA injection-produced CPA.

Saline-injected rats given EA or sham EA showed no preference or aversion to either compartment (Fig. 3, columns $3 \& 4$ ).

\section{Effects of an rACC opioid receptor antagonist injection on EA inhibition of CFA-induced CPA}

We injected CTOP, a $\mu$-selective opioid receptor antagonist, and nor-BNI, a $\kappa$-selective opioid receptor antagonist, into the rACC in saline- or CFA-injected rats given EA or sham. As shown in Fig. 4A, saline-injected rats given sham EA plus CTOP or nor-BNI showed no place avoidance or preference. Since saline injection and sham EA did not produced any CPA behavior (Fig. 3, column 3), the data indicate that the antagonist infusion per se did not produce any aversion. In contrast, CFA-injected rats given sham EA plus CTOP or nor-BNI showed significant aversion to a CFA-paired chamber (Fig. 4B). Since the degree of CPA was similar in CFA + sham + antagonist and CFA + sham + vehicle groups (Fig. 4B), the data suggest that the CFA injection induced little activation of endogenous opioid receptors in the rACC. Saline-injected rats given EA plus CTOP or nor-BNI also showed no CPA behavior (Fig. 4C).

As shown in Fig. 4D, CTOP rats, compared to saline rats, showed no significant aversive response to a pain-paired compartment, indicating that CTOP does not block EA inhibition of CPA. Nor-BNI rats showed significant aversive response to a pain-paired compartment compared to saline rats, indicating that nor-BNI does block EA inhibition of CPA. These results suggest $\mathrm{x}$-opioid receptors in the rACC are involved in the EA-produced inhibition of CPA.

\section{Histology}

Cannula and injection sites were located in the rACC as shown in Fig. 5. 


\section{Discussion}

\section{CFA-injection into the hind paw induces the affective dimension of pain}

In the present study, a CFA injection induced an avoidance response to a pain-paired compartment compared to saline injection, indicating that CFA produces the affective dimension of pain. Pain consists of sensory discrimination and negative affective components. Although the sensory component of pain has been studied extensively [14, 15], the affective component is not well understood. It has been reported that a formalin injection induces the affective dimension of pain [1]. Recent study also showed that suprathreshold mechanical stimulation of the paw ipsilateral to carrageenan-caused tissue injury induces significant aversion to a pain-paired environment [9]. These data demonstrate that affective response is a common phenomenon in inflammatory pain animal models and may be assessed with the CPA paradigm.

\section{EA inhibits negative affective response}

The present study demonstrates that EA treatment significantly inhibits the CFA-injectioncaused affective response. EA inhibition of CPA in this model is not due to a motor disorder: saline-injected rats given EA treatment showed no avoidance or preference to either of two compartments, indicating that EA treatment does not disrupt motor ability in rats. Moreover, since it has been reported that EA treatment does not cause the deterioration of memory [16], the EA inhibition of CPA is not the result of memory failure. Finally, as previously mentioned, acupuncture improved the affective dimension in patients with various chronic pain conditions in an uncontrolled observation [8]. Taken together, these studies indicate that acupuncture may be useful for treating the affective dimension of pain. Further, since EA completely eliminates the affective response but only partially alleviates the sensory component of pain in the CFA-inflammatory pain rat model [7], we believe that the affective component of pain is more sensitive to EA treatment than is the sensory component. This is consistent with the fact that low dosage celecoxib, diclofenac, and duloxetine significantly inhibited the avoidance response associated with stimulation of an injured paw, whereas higher dosages of these drugs were needed to attenuate mechanical allodynia assessed with von Frey filaments [3].

Additionally, it has been reported that acupuncture attenuated nicotine withdrawal-[17], maternal separation- [18], and chronic corticosterone-induced [19] anxiety-like behavior assessed with an elevated plus maze. These data and our own show that acupuncture may modulate mood disorders induced by a variety of factors.

\section{Opioids in the rACC are involved in EA inhibition of CPA}

Previous studies provide evidence that acupuncture treatment increases opioid levels in the brain, cerebral spinal fluid, and plasma [11,20], and that EA-produced analgesia is mediated by the endogenous opioid system [11, 13, 21]. It has been reported that the ACC plays a role in affective behavior related to pain. For instance, bilateral ACC lesions decrease paininduced escape/ avoidance behavior in rats $[1,10]$. In our study, nor-BNI infusion into the rACC before EA treatment blocked EA inhibition of CPA, while in contrast, CTOP pretreatment did not. These data suggest that EA inhibits CPA through rACC $\kappa$-opioid receptors.

It is known that opioid systems interact with glutamatergic transmission [22]. In a previous study, intra-ACC microinjection of 2-amino-5-phosphonovalerate (AP5), an N-methyl daspartate receptor (NMDAR) antagonist, inhibited formalin-induced affective behavior related to pain [23]. That suggests that NMDAR in the ACC is involved in the affective component of pain. Dynorphin A (DynA), an endogenous agonist of $\kappa$-opioid receptors, has 
been reported to interact with the NMDAR to block NMDA-activated currents [22]. Collectively, these studies suggest that endogenous opioids may impede NMDAR function to inhibit the affective dimension of pain.

In conclusion, the present study provides direct evidence that $10 \mathrm{~Hz}$ EA inhibits the spontaneous pain-induced affective response by activating $\mathrm{\kappa}$-opioid receptors in the rACC. Thus, EA benefits both the sensory and the affective dimensions of pain. The reciprocal effect between persistent pain and mood disorders is an extremely important issue given the prevalence of co-morbid emotional disorders in patients with chronic pain [24]. The results of this study provide a promising therapeutic approach, i.e. low-frequency EA, for the treatment of both the sensory-discriminative and the emotional-affective dimensions of pain.

\section{Acknowledgments}

This publication was made possible by grant number R21AT005474-01 and P01AT002605 from the National Center for Complementary and Alternative Medicine (NCCAM) at the National Institutes of Health. We would like to thank Dr. Lyn Lowry for her editorial support.

\section{References}

1. Johansen JP, Fields HL, Manning BH. The affective component of pain in rodents: Direct evidence for a contribution of the anterior cingulate cortex. Proc Natl Acad Sci U S A. 2001; 98:8077-8082. [PubMed: 11416168]

2. LaBuda CJ, Fuchs PN. Low dose aspirin attenuates escape/avoidance behavior, but does not reduce mechanical hyperalgesia in a rodent model of inflammatory pain. Neurosci Lett. 2001; 304:137140. [PubMed: 11343821]

3. Boyce-Rustay JM, Zhong C, Kohnken R, Baker SJ, Simler GH, Wensink EJ, Decker MW, Honore P. Comparison of mechanical allodynia and the affective component of inflammatory pain in rats. Neuropharmacology. 2010; 58:537-543. [PubMed: 19703478]

4. Cheng, X. Chinese Acupuncture and Moxibustion. Foreign Languages Press; Beijing: 1999.

5. Koo ST, Park YI, Lim KS, Chung K, Chung JM. Acupuncture analgesia in a new rat model of ankle sprain pain. Pain. 2002; 99:423-431. [PubMed: 12406517]

6. Zhang Y-Q, Ji G-C, Wu G-C, Zhao Z-Q. Excitatory amino acid receptor antagonists and electroacupuncture synergetically inhibit carrageenan-induced behavioral hyperalgesia and spinal fos expression in rats. Pain. 2002; 99:525-535. [PubMed: 12406529]

7. Lao L, Zhang R-X, Zhang G, Wang X, Berman BM, Ren K. A parametric study of electroacupuncture on persistent hyperalgesia and Fos protein expression in rats. Brain Res. 2004; 1020:18-29. [PubMed: 15312783]

8. Hammes MG, Flatau B, Bäcker M, Ehinger S, Conrad B, Tölle TR. Investigations on the effect of acupuncture on affective and sensory components of pain in patients with different stages of chronic pain. Schmerz. 2002; 16:103-13. [PubMed: 11956895]

9. Hummel M, Lu P, Cummons TA, Whiteside GT. The persistence of a long-term negative affective state following the induction of either acute or chronic pain. Pain. 2008; 140:436-445. [PubMed: 18945547]

10. LaGraize SC, Labuda CJ, Rutledge MA, Jackson RL, Fuchs PN. Differential effect of anterior cingulate cortex lesion on mechanical hypersensitivity and escape/avoidance behavior in an animal model of neuropathic pain. Exp Neurol. 2004; 188:139-48. [PubMed: 15191810]

11. Mayer DJ. Biological mechanisms of acupuncture. Prog Brain Res. 2000; 122:457-77. [PubMed: 10737077]

12. Fenu S, Spina L, Rivas E, Longoni R, Di Chiara G. Morphine-conditioned single-trial place preference: role of nucleus accumbens shell dopamine receptors in acquisition, but not expression. Psychopharmacology. 2006; 187:143-53. [PubMed: 16724186] 
13. Zhang RX, Lao L, Wang L, Liu B, Wang X, Ren K, Berman BM. Involvement of opioid receptors in electroacupuncture-produced anti-hyperalgesia in rats with peripheral inflammation. Brain Res. 2004; 1020:12-17. [PubMed: 15312782]

14. Millan MJ. Descending control of pain. Prog Neurobiol. 2002; 66:355-474. [PubMed: 12034378]

15. Heinricher MM, Tavares I, Leith JL, Lumb BM. Descending control of nociception: Specificity, recruitment and plasticity. Brain Res Rev. 2009; 60:214-25. [PubMed: 19146877]

16. Chen J-H, Liang J, Wang G-b, Han J-S, Cui C-1. Repeated 2 Hz peripheral electrical stimulations suppress morphine-induced CPP and improve spatial memory ability in rats. Exp Neurol. 2005; 194:550-556. [PubMed: 15890338]

17. Chae Y, Yeom M, Han JH, Park HJ, Hahm DH, Shim I, Lee HS, Lee H. Effect of acupuncture on anxiety-like behavior during nicotine withdrawal and relevant mechanisms. Neurosci Lett. 2008; 430:98-102. [PubMed: 18060697]

18. Park HJ, Chae Y, Jang J, Shim I, Lee H, Lim S. The effect of acupuncture on anxiety and neuropeptide $\mathrm{Y}$ expression in the basolateral amygdala of maternally separated rats. Neurosci Lett. 2005; 377:179-184. [PubMed: 15755522]

19. Lee B, Shim I, Lee HJ, Yang Y, Hahm DH. Effects of acupuncture on chronic corticosteroneinduced depression-like behavior and expression of neuropeptide $\mathrm{Y}$ in the rats. Neurosci Lett 2009. 2009; 453:151-156.

20. Cao X. Scientific bases of acupuncture analgesia. Acupunct Electrother Res. 2002; 27:1-14. [PubMed: 12044016]

21. Han J-S. Acupuncture. neuropeptide release produced by electrical stimulation of different frequencies. Trends Neurosci. 2003; 26:17-22. [PubMed: 12495858]

22. Kanemitsu Y, Hosoi M, Zhu PJ, Weight FF, Peoples RW, McLaughlin JS, Zhang L. Dynorphin A inhibits NMDA receptors through a pH-dependent mechanism. Mol Cell Neurosci. 2003; 24:52537. [PubMed: 14664805]

23. Lei L-G, Sun S, Gao Y-J, Zhao Z-Q, Zhang Y-Q. NMDA receptors in the anterior cingulate cortex mediate pain-related aversion. Exp Neurol. 2004; 189:413-421. [PubMed: 15380491]

24. McWilliams LA, Cox BJ, Enns MW. Mood and anxiety disorders associated with chronic pain: an examination in a nationally representative sample. Pain. 2003; 106:127-133. [PubMed: 14581119] 


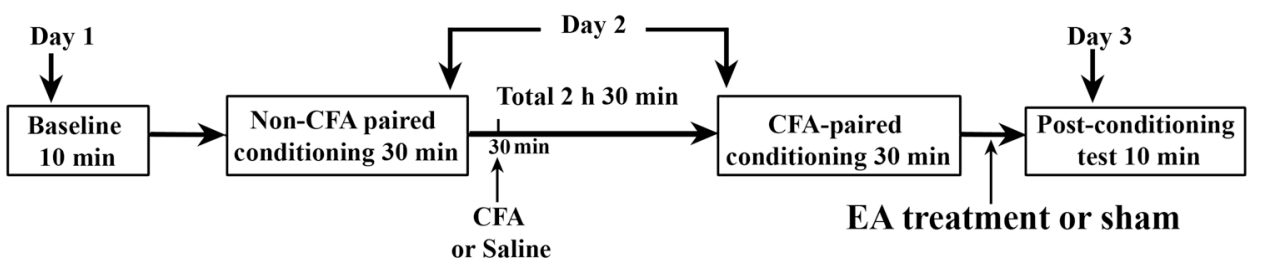

Fig. 1.

Flow chart of the experimental design. On day one, time spent by the rats in each of two distinct compartments was recorded during a 10-min preconditioning period. On day two, each rat freely explored the first compartment for $30 \mathrm{~min}$ before a CFA injection and the second compartment for $30 \mathrm{~min}$ after CFA. On day three, after EA treatment, the time spent in each compartment during a 10-min post-conditioning test was recorded again. 


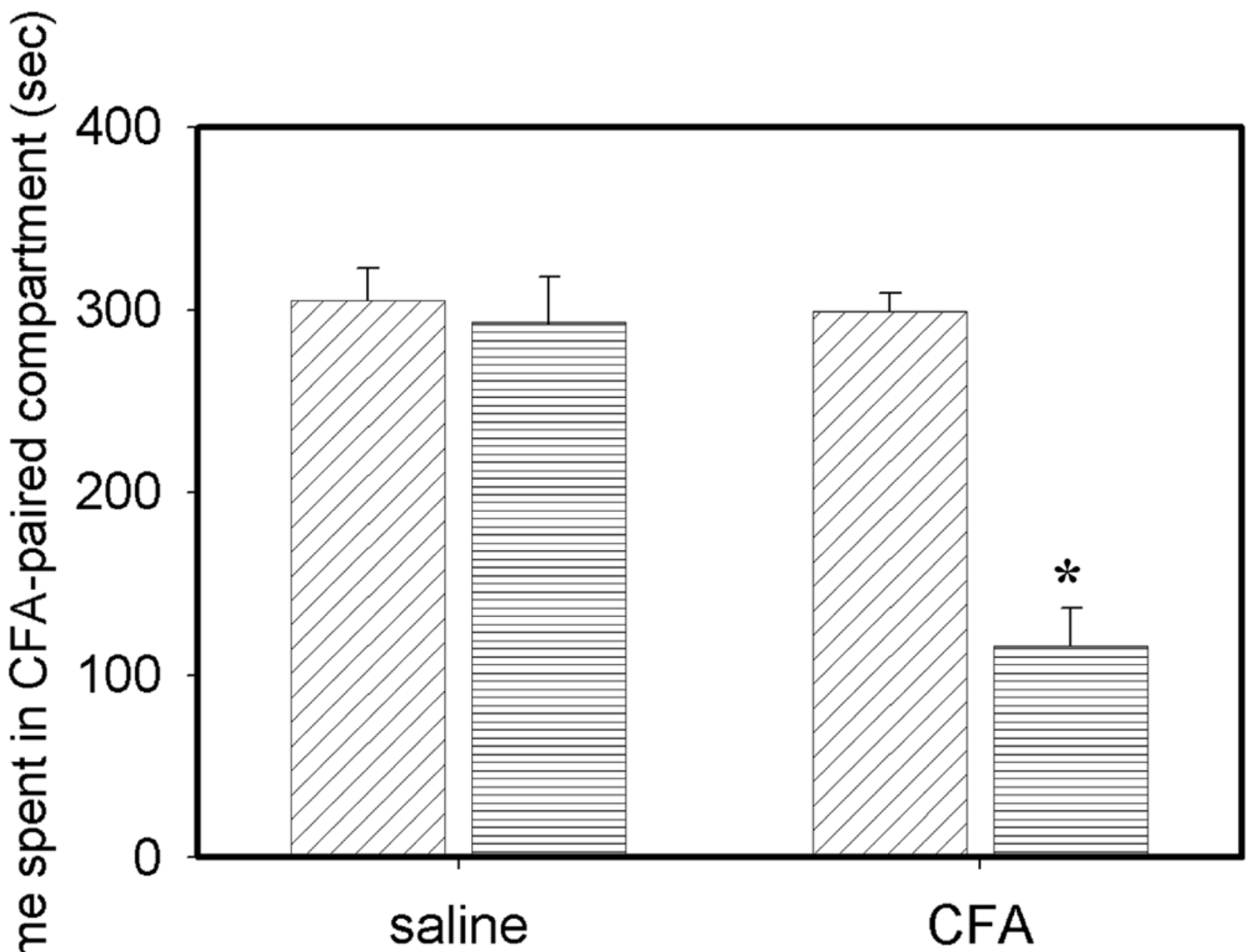

Fig. 2.

CFA injection into one hind paw induced conditioned place avoidance ( $n=6$ per group). Saline-injection controls showed no preference or aversion to either chamber. CFA-injected rats showed aversive responses to the CFA-paired chamber during the postconditioning test. Data are presented as mean \pm se. $* \mathrm{P}<0.05$ vs pre-conditioning test. 


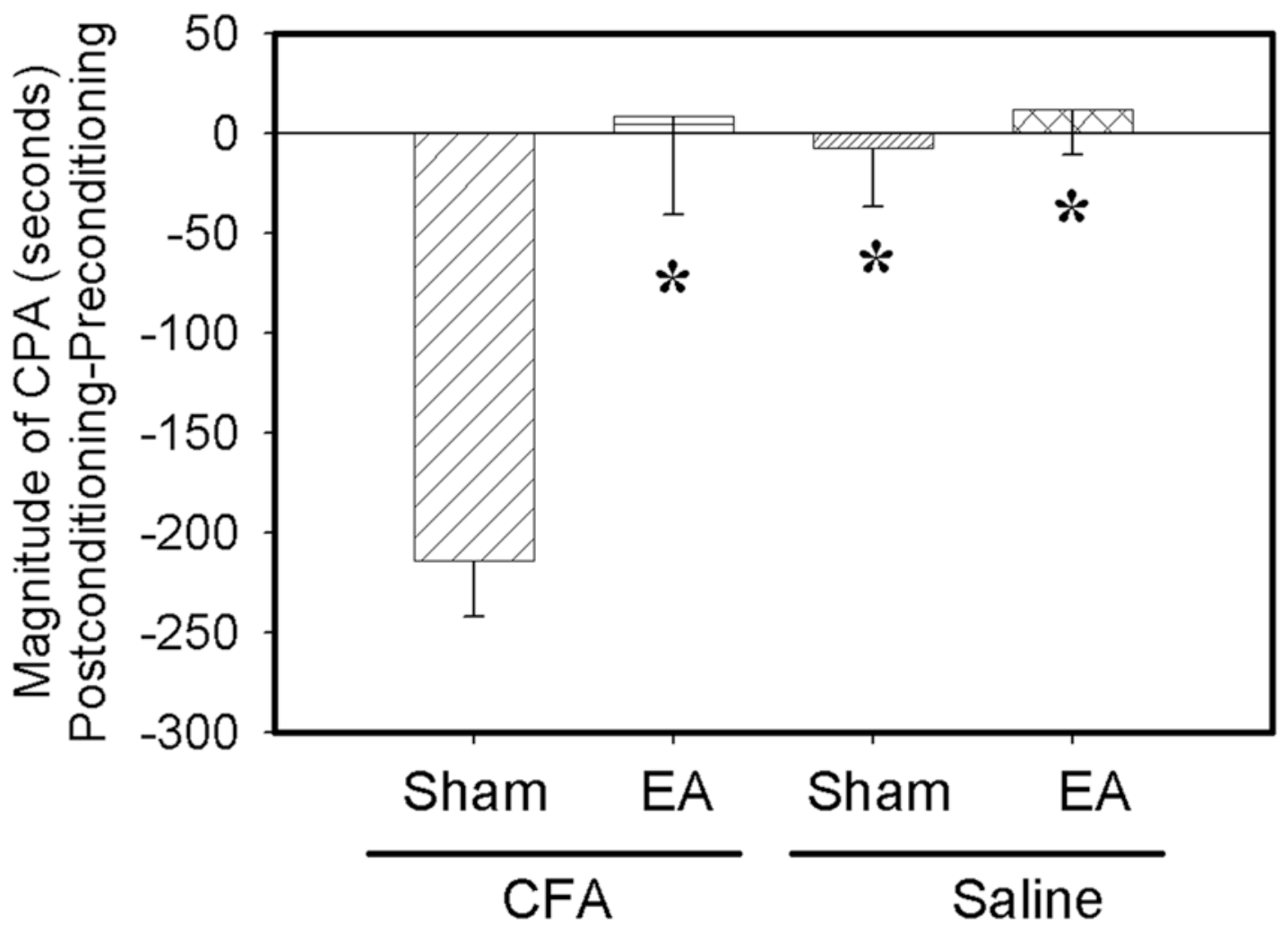

Fig. 3.

Columns $1 \& 2$ EA inhibition of CFA-induced affective response ( $n=8$ per group). EA was given on day three before post-conditioning test. CFA-injected rats given sham EA showed avoidance to a pain-paired compartment. EA treatment inhibited the avoidance response. $* \mathrm{P}<0.05$ compared to sham control. Columns 3 \& 4: Conditioned place preference score in saline-injected rats with EA or sham EA ( $n=6$ per group), neither of which produced preference or avoidance of the respectively paired compartment. $* \mathrm{P}<0.05$ compared to sham control in CFA-injected rats. 


\section{A: saline + sham EA + antagonist $B$ : CFA + sham EA +antagonist}
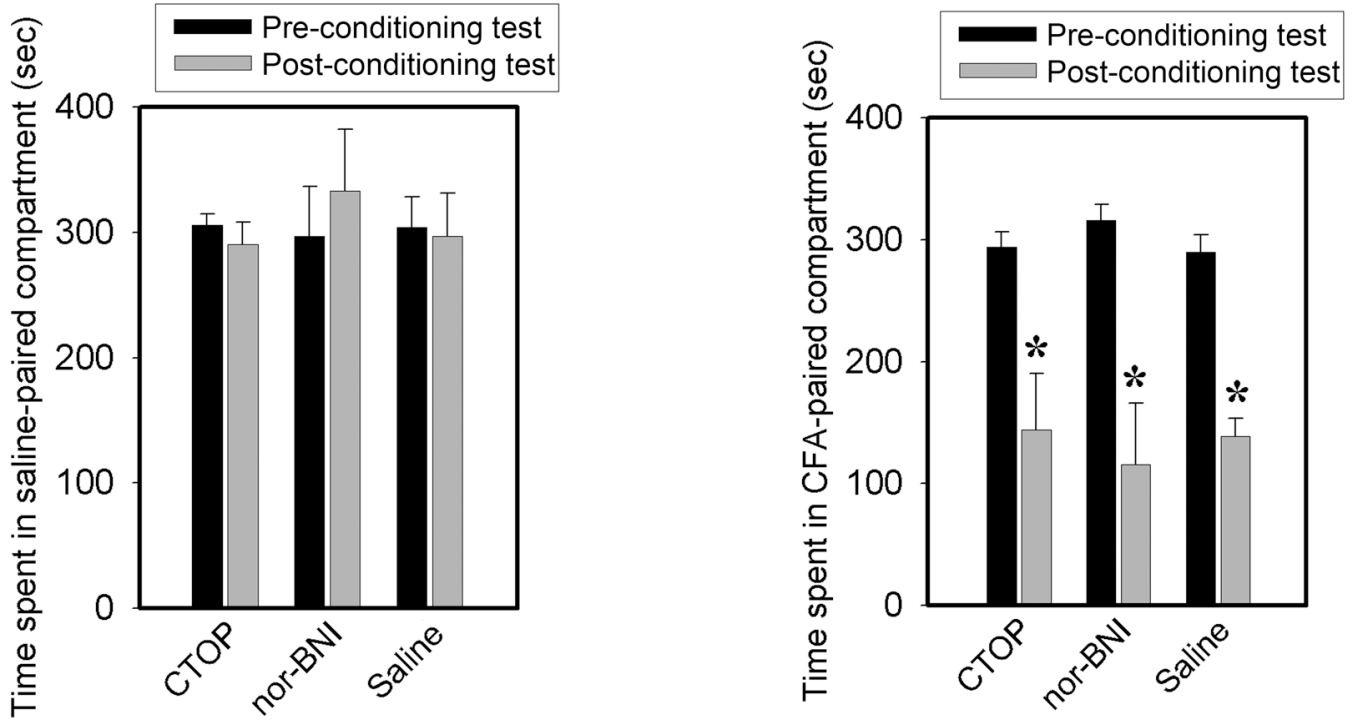

\section{C: saline + EA + antagonist}

$D: C F A+E A+$ antagonist
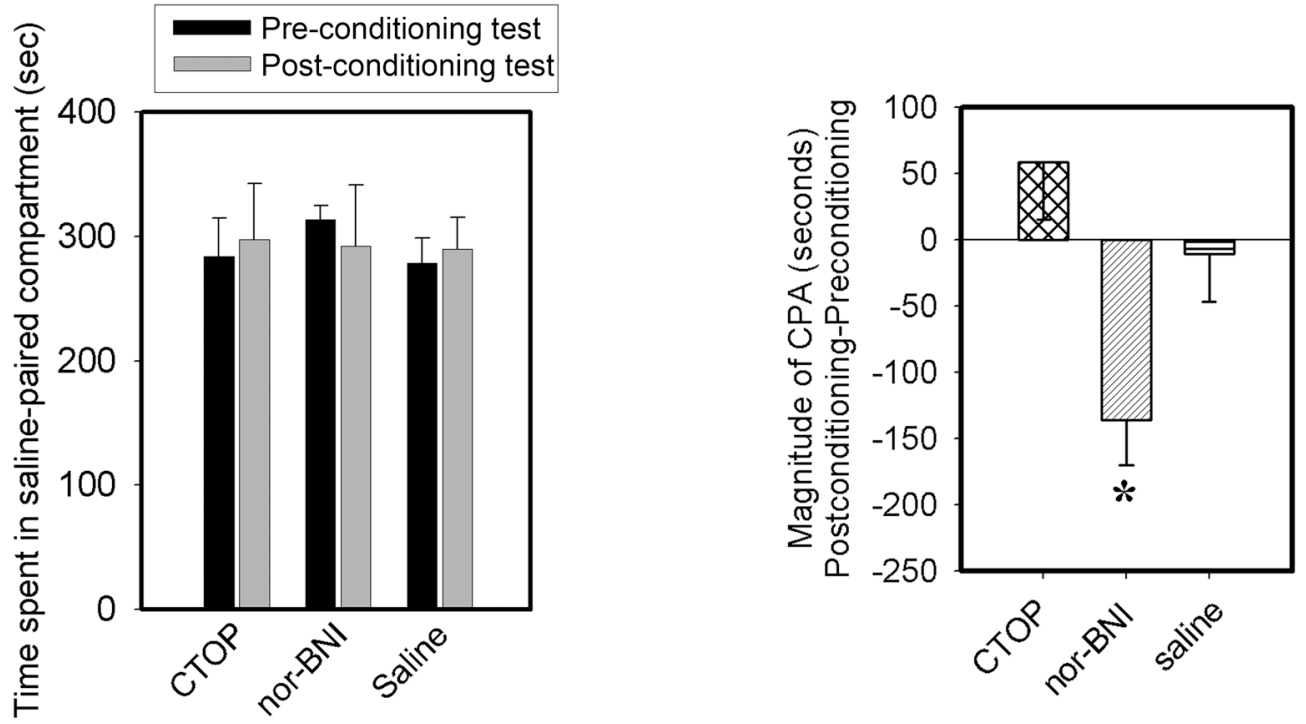

Fig. 4.

Effects of $\mu$ and $\kappa$ opioid receptors on EA action. 4A: Antagonists, CTOP for $\mu$ and norBNI for $\kappa$, were infused into the rACC in saline-injected, sham EA-treated rats. The rats showed no CPA. 4B. Antagonists were infused in CFA-injected, sham EA-treated rats. The rats showed significant $\mathrm{CPA} .{ }^{*} \mathrm{P}<0.05$ vs pre-conditioning test results. $4 \mathrm{C}$. Antagonists were infused in saline-injected, EA-treated rats. The rats showed no CPA. 4D: The effect of opioid receptor antagonists on EA inhibition of CFA-induced affective response. Nor-BNI blocked the EA inhibition of the affective response, but CTOP did not. ${ }^{*} \mathrm{P}<0.05$ compared to saline control. 

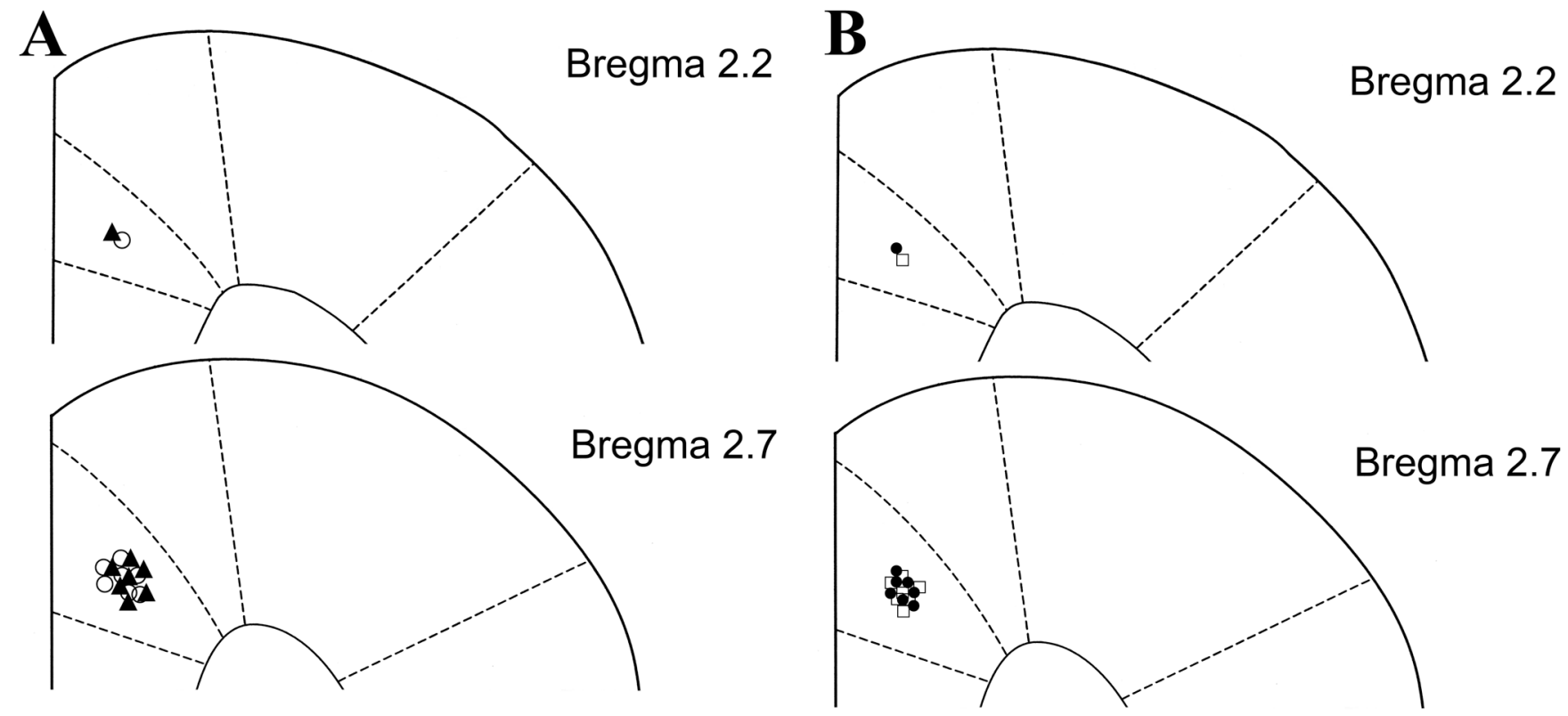

\section{$\Delta:$ saline + sham EA + antagonist $\square:$ CFA + EA + antagonist $\circ:$ CFA + sham EA + antagonist $\bullet$ : saline + EA + antagonist}

Fig. 5.

Coronal brain section reconstructions showing microinjection sites and cannula placement in the rACC. Each symbol represents one rat. 